\title{
The fréchet stress-strength model
}

\author{
Salah H Abid \\ Mathematics department, Education college, Al-Mustansiriya University, Baghdad, Iraq \\ E-mail: abidsalah@gmail.com
}

Copyright $($ ) 2014 Salah H Abid. This is an open access article distributed under the Creative Commons Attribution License, which permits unrestricted use, distribution, and reproduction in any medium, provided the original work is properly cited.

\begin{abstract}
This paper deals with the determination of $\mathrm{R}=\mathrm{P}[\mathrm{Y}<\mathrm{X}]$ when $\mathrm{X}$ and $\mathrm{Y}$ are two independent Fréchet distributions with different scale parameters and different shape parameters. Special cases when $\mathrm{X}$ and $\mathrm{Y}$ have the same shape parameter but having the different scale parameters, and when $\mathrm{X}$ and $\mathrm{Y}$ have the same scale parameter but having the different shape parameters, are also considered. The divergence problem of $\mathrm{R}$ is also discussed. Different methods to estimate $\mathrm{R}$ and Fréchet distribution parameters are studied, Maximum Likelihood estimator, Moments estimator, Regression estimator, Percentile estimator, least square estimator and L-moments estimator. An empirical study was conducted to support the theoretical aspect.
\end{abstract}

Keywords: Fréchet Distributions, Percentile Estimator, L-Moments Estimator, Maximum Likelihood Estimator, Stress- Strength

\section{Introduction}

Inferences about $\mathrm{R}=\mathrm{P}[\mathrm{Y}<\mathrm{X}]$, where $\mathrm{X}$ and $\mathrm{Y}$ are two independent random variables, is very common in the reliability literature. For example, if $\mathrm{X}$ is the strength of a component which is subject to a stress $\mathrm{Y}$, then $\mathrm{R}$ is a measure of system performance and arises in the context of mechanical reliability of a system. The system fails if and only if at any time the applied stress is greater than its strength.

It is well known that the probability density function of the Fréchet random variable $\mathrm{W}$ is,

$\mathrm{f}(\mathrm{w})=\mathrm{ab} \mathrm{w}^{-(\mathrm{b}+1)} \mathrm{e}^{-\mathrm{a} \mathrm{w}} \quad, 0<w<\infty$

Where $a>0$ is the scale parameter and $b>0$ is the shape parameter.

The Fréchet distribution is a special case of the generalized extreme value distribution. It has the cumulative distribution function,

$\mathrm{F}(\mathrm{w})=\mathrm{e}^{-\mathrm{a} \mathrm{w}^{-\mathrm{b}}}$

And has standardized moment,

$E\left(w^{r}\right)=a^{r / b} \Gamma\left(\frac{b-r}{b}\right)$

In this paper we will refer to the above distribution by $\mathrm{W} \sim \operatorname{Fr}(\mathrm{a}, \mathrm{b})$, which is mean that the random variable $\mathrm{W}$ follow Fréchet distribution with parameters $\mathrm{a}$ and $\mathrm{b}$.

\section{Stress-strength reliability}

Let $\mathrm{Y}$ and $\mathrm{X}$ be the stress and the strength random variables, independent of each other, follow respectively $\operatorname{Fr}(\mu, \alpha)$ and $\operatorname{Fr}(\lambda, \theta)$, then,

$\mathrm{R}=\mathrm{P}(\mathrm{Y}<X)=\int_{\mathrm{x}=0}^{\infty} \mathrm{f}_{\mathrm{X}}(\mathrm{x}) \mathrm{F}_{\mathrm{Y}}(\mathrm{x}) \mathrm{dx}$

$=\int_{\mathrm{x}=0}^{\infty} \lambda \theta \mathrm{x}^{-(\theta+1)} \mathrm{e}^{-\lambda \mathrm{x}^{-\theta}} \mathrm{e}^{-\mu \mathrm{x}^{-\alpha}} \mathrm{dx}$

Now, let $Z=X^{-\theta} \rightarrow X=Z^{-\frac{1}{\theta}}, d x=\frac{-1}{\theta} z^{-\left(\frac{1}{\theta}+1\right)} d z$, then,

$\mathrm{R}=\lambda \int_{0}^{\infty} \mathrm{e}^{-\lambda \mathrm{z}} \mathrm{e}^{-\mu \mathrm{z}^{\alpha / \theta}} \mathrm{dz}$ 


$$
\begin{aligned}
& =\lambda \int_{0}^{\infty} \mathrm{e}^{-\lambda \mathrm{z}} \sum_{\mathrm{k}=0}^{\infty} \frac{\left(-\mu \mathrm{z}^{\alpha / \theta}\right)^{\mathrm{k}}}{\mathrm{k} !} \mathrm{dz} \\
& =\lambda \sum_{\mathrm{k}=0}^{\infty} \frac{(-\mu)^{\mathrm{k}}}{\mathrm{k} !} \int_{0}^{\infty} \mathrm{e}^{-\lambda \mathrm{z}} \mathrm{z}^{\alpha \mathrm{k} / \theta} \mathrm{dz} \\
& =\lambda \sum_{\mathrm{k}=0}^{\infty} \frac{(-\mu)^{\mathrm{k}}}{\mathrm{k} !} \Gamma\left(\frac{\alpha \mathrm{k}}{\theta}+1\right)(1 / \lambda)^{\alpha} \\
& =\lambda^{-\alpha+1} \sum_{\mathrm{k}=0}^{\infty} \frac{(-\mu)^{\mathrm{k}}}{\Gamma(\mathrm{k}+1)} \Gamma\left(\frac{\alpha \mathrm{k}}{\theta}+1\right)
\end{aligned}
$$

As special cases,

(1) If $\mathrm{X}$ and $\mathrm{Y}$ have the same shape parameter $(\alpha=\theta)$ but having the different scale parameters then, $\mathrm{R}=\frac{\lambda}{\lambda+\mu}$

(2) If $\mathrm{X}$ and $\mathrm{Y}$ have the same scale parameter $(\mu=\lambda)$ but having the different shape parameters then,

$$
\mathrm{R}=\sum_{\mathrm{k}=0}^{\infty} \frac{(-1)^{\mathrm{k}}}{\Gamma(\mathrm{k}+1)} \lambda^{\mathrm{k}-\alpha+1} \Gamma\left(\frac{\alpha \mathrm{k}}{\theta}+1\right)
$$

\section{The divergence problem}

In this section we will check and discuss the convergence of the alternating series in (4). At first, let us rewrite the series in (4) as,

$$
\begin{aligned}
& \mathrm{R}=\lambda^{-\alpha+1} \sum_{\mathrm{k}=0}^{\infty} \frac{(-\mu)^{\mathrm{k}}}{\Gamma(\mathrm{k}+1)} \Gamma\left(\frac{\alpha \mathrm{k}}{\theta}+1\right) \\
& =\lambda^{-\alpha+1} \sum_{\mathrm{j}=0}^{\infty}(-1)^{\mathrm{j}} \mathrm{u}_{\mathrm{j}}
\end{aligned}
$$

Where, $\mathrm{u}_{\mathrm{j}}=\frac{(\mu)^{\mathrm{j}}}{\Gamma(\mathrm{j}+1)} \Gamma\left(\frac{\alpha \mathrm{j}}{\theta}+1\right) \cong \prod_{\mathrm{r}=0}^{\mathrm{b}}\left(\frac{\alpha}{\theta} \mathrm{j}-\mathrm{r}\right)(\mu)^{\mathrm{j}}$, Since,

$\Gamma\left(\frac{\alpha}{\theta} \mathrm{j}+1\right)=\prod_{\mathrm{r}=0}^{\mathrm{b}}\left(\frac{\alpha}{\theta} \mathrm{j}-\mathrm{r}\right) \Gamma(\mathrm{j}+1)$

Where, $b=j\left(\frac{\alpha}{\theta}-1\right)-1$.

So, by using Leibniz test [ 1 ], which is based on satisfying the following two conditions,

i. $\quad u_{j+1} \leq u_{j}, \forall j \in N$

ii. $\quad \lim _{j \rightarrow \infty} u_{j}=0$

To be the series in (4) converges, one can get

(a) Let , $\delta(j)=\frac{u_{j+1}}{u_{j}}$, so $\delta(j) \leq 1$ is equivalent to $u_{j+1} \leq u_{j}$, then by using (7) and putting

$$
\begin{aligned}
\mathrm{d}= & (\mathrm{j}+1)\left(\frac{\alpha}{\theta}-1\right)-1, \\
\delta(j) & =\frac{(\mu)^{\mathrm{j}+1} \prod_{\mathrm{r}=0}^{\mathrm{d}}\left(\frac{\alpha}{\theta}(\mathrm{j}+1)-\mathrm{r}\right)}{(\mu)^{\mathrm{j}} \prod_{\mathrm{r}=0}^{\mathrm{b}}\left(\frac{\alpha}{\theta} \mathrm{j}-\mathrm{r}\right)} \\
& =\frac{\mu \alpha}{\theta} \prod_{\mathrm{k}=1}^{\frac{\alpha}{\theta}-1}\left(\frac{\alpha}{\theta}(\mathrm{j}+1)-\mathrm{k}\right)
\end{aligned}
$$

It is clear that $\delta(j)$ cannot be less than or equal to one (or equivalently $\mathrm{u}_{\mathrm{j}+1}$ cannot be less than or equal to $\mathrm{u}_{\mathrm{j}}$ ), except for $\alpha<\theta$.

(b) It is clear that also, $\lim _{\mathrm{j} \rightarrow \infty} \prod_{\mathrm{r}=0}^{\mathrm{b}}\left(\frac{\alpha}{\theta} \mathrm{j}-\mathrm{r}\right)(\mu)^{\mathrm{j}} \neq 0$ except for some rare values of $\alpha<\theta$ and $\mu$.

Generally, it can be said that the series in (4) is convergent provided $\alpha<\theta$, otherwise, we can use the relation $\mathrm{R}=\mathrm{P}[\mathrm{Y}<\mathrm{X}]=1-\mathrm{P}[\mathrm{X} \leq \mathrm{Y}]$. So the final formula to get $\mathrm{R}$ is,

$$
\mathrm{R}= \begin{cases}\lambda^{-\alpha+1} \sum_{\mathrm{k}=0}^{\infty} \frac{(-\mu)^{\mathrm{k}}}{\Gamma(\mathrm{k}+1)} \Gamma\left(\frac{\alpha \mathrm{k}}{\theta}+1\right) & \text { if } \alpha<\theta \\ 1-\mu^{-\theta+1} \sum_{\mathrm{k}=0}^{\infty} \frac{(-\lambda)^{\mathrm{k}}}{\Gamma(\mathrm{k}+1)} \Gamma\left(\frac{\theta \mathrm{k}}{\alpha}+1\right) & \text { if } \theta<\alpha\end{cases}
$$

\section{Parameters estimation of fréchet distribution}

The main aim of this section is to study different estimators of the unknown parameters of a Fréchet distribution,

1. Maximum Likelihood estimators (MLE).

If $\mathrm{y}_{1}, \mathrm{y}_{2}, \ldots, \mathrm{y}_{\mathrm{n}}$ is a random sample from $\operatorname{Fr}(\mathrm{a}, \mathrm{b})$, then the log-likelihood function is

$\mathrm{L}(\mathrm{a}, \mathrm{b})=\mathrm{n} \operatorname{Ln}(\mathrm{a})+\mathrm{n} \operatorname{Ln}(\mathrm{b})-(\mathrm{b}+1) \sum_{\mathrm{i}=1}^{\mathrm{n}} \operatorname{Ln}\left(\mathrm{y}_{\mathrm{i}}\right)-\mathrm{a} \sum_{\mathrm{i}=1}^{\mathrm{n}} \mathrm{y}_{\mathrm{i}}^{-\mathrm{b}}$

The normal equations become,

$\frac{\partial \mathrm{L}}{\partial \mathrm{a}}=\frac{\mathrm{n}}{\mathrm{a}}-\sum_{\mathrm{i}=1}^{\mathrm{n}} \mathrm{y}_{\mathrm{i}}^{-\mathrm{b}}$

$\frac{\partial \mathrm{L}}{\partial \mathrm{b}}=\frac{\mathrm{n}}{\mathrm{b}}-\sum_{\mathrm{i}=1}^{\mathrm{n}} \operatorname{Ln}\left(\mathrm{y}_{\mathrm{i}}\right)+\mathrm{ab} \sum_{\mathrm{i}=1}^{\mathrm{n}} \mathrm{y}_{\mathrm{i}}^{-(\mathrm{b}+1)}$ 
From (11) we obtain the MLE of a as a function of $b$, say, $\hat{a}(b)$ where,

$\hat{\mathrm{a}}(\mathrm{b})=\frac{\mathrm{n}}{\sum_{\mathrm{i}=1}^{\mathrm{n}} \mathrm{y}_{\mathrm{i}}^{-\mathrm{b}}}$

Putting $\hat{a}(b)$ in (10), we obtain,

$g(b)=C+n \operatorname{Ln}\left(\frac{b}{\sum_{i=1}^{n} y_{i}^{-b}}\right)-(b+1) \sum_{i=1}^{n} \operatorname{Ln}\left(y_{i}\right)$

Therefore, MLE of $b$, say $\hat{b}_{M L E}$, can be obtained by maximizing (14) with respect to $b$. Very simple iterative procedure can be used to find a solution of (14) and it works very well. Once we obtain $\hat{b}_{M L E}$, the MLE of $a$, say $\hat{a}_{M L E}$ can be obtained from (13) as $\hat{a}_{M L E}=\hat{a}\left(\hat{b}_{M L E}\right)$.

Now, we state the asymptotic normality results,

$\left[\sqrt{n}\left(\hat{a}_{M L E}-a\right), \sqrt{n}\left(\hat{b}_{M L E}-b\right)\right] \rightarrow N_{2}\left(\underline{0}, I^{-1}(a, b)\right)$

Where,

$I(a, b)=\frac{-1}{n}\left(\begin{array}{cc}E \frac{\partial^{2} L}{\partial a^{2}} & E \frac{\partial^{2} L}{\partial a \partial b} \\ E \frac{\partial^{2} L}{\partial a \partial b} & E \frac{\partial^{2} L}{\partial b^{2}}\end{array}\right)$ Is the Fisher information matrix.

The elements of Fisher information matrix are as follows,

$\frac{\partial^{2} L}{\partial a^{2}}=\frac{-n}{a^{2}}$

$\frac{\partial^{2} L}{\partial b^{2}}=\frac{-n}{b^{2}}-a b(b+1) \sum_{i=1}^{n} y_{i}^{-(b+2)}+a \sum_{i=1}^{n} y_{i}^{-(b+1)}$

$=\frac{-n}{b^{2}}-n b(b+1) \frac{\Gamma\left(\frac{2}{b}+2\right)}{a^{\left(\frac{2}{b}-1\right)}}+n \frac{\Gamma\left(\frac{1}{b}+2\right)}{a^{\left(\frac{1}{b}-1\right)}}$

$\frac{\partial^{2} L}{\partial a \partial b}=b \sum_{i=1}^{n} y_{i}^{-(b+1)}$

$$
=n b \frac{\Gamma\left(\frac{1}{b}+2\right)}{a\left(\frac{1}{b}\right)}
$$

2. The exact estimators of moments Method (EMME).

Here we provide the method of moments estimators of the parameters of a Fréchet distribution when both are unknown. If $Y$ follows $\operatorname{Fr}(a, b)$, then

$E(y)=a^{1 / b} \Gamma(1-1 / b)$

$\operatorname{Var}(y)=a^{2 / b} \Gamma(1-2 / b)-a^{2 / b} \Gamma^{2}(1-1 / b)$

And then the coefficient of variation is,

$C v=\frac{\sqrt{\operatorname{Var}(y)}}{E(y)}=\frac{\sqrt{\Gamma(1-2 / b)-\Gamma^{2}(1-1 / b)}}{\Gamma(1-1 / b)}$

The $C v$ is independent of the scale parameter $a$. Therefore equating the sample $C v$ with the Population $C v$, we obtain

$\frac{s}{\bar{y}}=\frac{\sqrt{\Gamma(1-2 / b)-\Gamma^{2}(1-1 / b)}}{\Gamma(1-1 / b)}$

Where $s^{2}=\sum_{i=1}^{n}\left(y_{i}-\bar{y}\right)^{2} /(n-1)$ and $\bar{y}=\sum_{i=1}^{n} y_{i} / n$.

We need to solve (19) to obtain the EMME of $b$, say $\hat{b}_{E M M E}$. once we estimate $b$, we can use (16) to obtain the EMME of a We need to use some iterative procedure to solve (19).

3. The approximate estimators of moments Method (AMME).

If $Y$ follows $\operatorname{Fr}(a, b)$, then the median and the mode of $Y$ are,

$M e=(a / \operatorname{Ln}(2))^{1 / b}$

$M o=(a b /(b+1))^{1 / b}$

Since,

$\operatorname{Ln}\left(\frac{M o}{M e}\right)=\frac{1}{b}\left[\operatorname{Ln}\left(\operatorname{Ln}(2)-\operatorname{Ln}\left(1+\frac{1}{b}\right)\right]\right.$

is independent of the scale parameter $a$, then, after calculating the sample mode and the sample median and substituting their values in (22), one can get the AMME of $b$, say, $\hat{b}_{A M M E}$ by solving iteratively (22). Once we estimate $b$, we can use (20) to obtain the AMME of $a$.

4. Estimators based on percentiles (PE).

Kao in (1959) originally explored this method by using the graphical approximation to the best linear unbiased estimators. The estimators can be obtained by fitting a straight line to the theoretical points obtained from the distribution function and the sample percentile points. In the case of a Fréchet distribution, it is possible to use the same concept to obtain the estimators of $a$ and $b$ based on percentiles because of the structure of its distribution function. Since $F(y)$ defined in (2), therefore,

$y=(-\operatorname{Ln}(F(y)) / a)^{-1 / b}$

If $p_{i}$ denotes some estimate of $F\left(y_{(i)} ; a, b\right)$ then the estimate of $a$ and $b$ can be obtained by minimizing,

$\left[y_{(i)}-\left(-\operatorname{Ln}\left(p_{i}\right) / a\right)^{-1 / b}\right]^{2}$ 
With respect to $a$ and $b$. (24) Is a nonlinear function of $a$ and $b$. It is possible to use some nonlinear Regression techniques to estimate $a$ and $b$ simultaneously. Actually, it is possible to use several $p_{i}{ }^{\prime}$ s as Estimators of $F\left(y_{(i)}\right)$. $\left.p_{i}=i /(n+1)\right)$ is the most used estimator of $F\left(y_{(i)}\right)$ since it is equal to $E\left(F\left(y_{(i)}\right)\right)$. We have also used this $p_{i}$ here.

For some other choices of $p_{i}{ }^{\prime}$ s, see Mann, Schafer and Singpurwalla (1974).

5. Least squares (LSE) and Weighted least squares (WLSE) estimators

This method was originally suggested by Swain, Venkatraman and Wilson (1988) to estimate the parameters of Beta distribution. Suppose $y_{1}, y_{2}, \ldots, y_{n}$ is a random sample of size $n$ from a distribution function $F($.$) and suppose y_{(i)}$ $(i=1,2, \ldots, n)$ Denotes the ordered sample. This method uses the distribution of $F\left(y_{(i)}\right)$. For a sample of Size $n$, we have [5],

$E\left(F\left(y_{(i)}\right)\right)=\frac{j}{n+1} \quad, \quad \operatorname{Var}\left(F\left(y_{(i)}\right)\right)=j(n-j+1) /\left((n+1)^{2}(n+2)\right)$ And

$\operatorname{Cov}\left[\left(F\left(y_{(i)}\right)\right),\left(F\left(y_{(k)}\right)\right)\right]=j(n-k+1) /\left((n+1)^{2}(n+2)\right)$ for $j<k$

So, one can obtain the LS estimators by minimizing, $\sum_{j=1}^{n}\left(F\left(y_{(i)}-j /(n+1)\right)^{2}\right.$ with respect to the unknown parameters. Therefore in the case of Fréchet distribution, the least squares estimators of $a$ and $b$, say $\hat{a}_{L S E}$ and $\hat{b}_{L S E}$ respectively, can be obtained by minimizing,

$\sum_{j=1}^{n}\left(\operatorname{Exp}\left(-\mathrm{a} y_{(j)}^{-b}\right)-j /(n+1)\right)^{2}$

With respect to $a$ and $b$.

The weighted least squares estimators of $a$ and $b$, say $\hat{a}_{W L S E}$ and $\hat{b}_{W L S E}$ respectively, can be obtained by minimizing,

$\sum_{j=1}^{n} w_{j}\left(\operatorname{Exp}\left(-a y_{(j)}^{-b}\right)-j /(n+1)\right)^{2}$

With respect to $a$ and $b$, where $w_{j}=1 / \operatorname{Var}\left(F\left(y_{(i)}\right)\right)=(n+1)^{2}(n+2) /(j(n-j+1))$.

6. L-Moment estimators (LME)

L-moments are expectations of certain linear combinations of order statistics. This method Originally suggested by Hosking (1990). L-Moments is similar to the method of moments in that We will be solving a system of equations whose order is equal to the number of parameters we are trying to estimate. However, the set of L-Moments equations is instead defined as

$\beta_{r}=E\left(Y F^{r}(Y)\right) \equiv \int_{-\infty}^{\infty} y F^{r}(y) f(y) d y=\int_{0}^{1} y(F) F^{r} d F$

Where $F(y)$ is the cumulative distribution function of the density function $f(y)$, as we defined before. We will set this equal to an unbiased estimate of $\beta_{r}$, which is defined as

$\mathrm{b}_{\mathrm{r}}=\frac{1}{n\left(\begin{array}{c}n-1 \\ r\end{array}\right)} \sum_{i=1}^{n}\left(\begin{array}{c}i-1 \\ r\end{array}\right) y_{(i)}$

Where $y_{(1)} \leq y_{(2)} \leq \cdots \leq y_{(n)}$ are the sorted values of the observations $y_{1}, y_{2}, \ldots, y_{n}$.

Now, If $Y$ follows $\operatorname{Fr}(a, b)$, then

$\beta_{r} \equiv \int_{0}^{\infty} y e^{-r a y^{-b}} a b y^{-(b+1)} e^{-a y^{-b}} d y=a b \int_{0}^{\infty} y y^{-(b+1)} e^{-a(r+1) y^{-b}} d y$

$=\frac{1}{r+1} \int_{0}^{\infty} a(r+1) b y y^{-(b+1)} e^{-a(r+1) y^{-b}} d y$

$=\frac{1}{r+1}(a(r+1))^{1 / b} \Gamma(1-1 / b)$

Which implies to, $\beta_{0}=a^{1 / b} \Gamma(1-1 / b)$ and $\beta_{1}=(2 a)^{1 / b} \Gamma(1-1 / b) / 2$.

Since, $\quad \mathrm{b}_{0}=\sum_{i=1}^{n} y_{i} / n=\bar{y}$ and $_{1}=\sum_{i=1}^{n}(i-1) y_{(i)} /(n(n+1))$, then by equating $\beta_{0}$ with $\mathrm{b}_{0}$ and $\beta_{1}$ with $\mathrm{b}_{1}$, we obtained the LM estimators of $a$ and $b$ as,

$\hat{b}_{L M E}=\left(\frac{\operatorname{Ln}\left(\mathrm{Б}_{1}\right)-\operatorname{Ln}\left(\mathrm{Б}_{0}\right)}{\operatorname{Ln}(2)}+1\right)^{-1}$ And $\hat{a}_{L M E}=\left(\frac{\mathrm{Б}_{0}}{\Gamma\left(1-1 / \hat{b}_{L M E}\right)}\right)^{\hat{b}_{L M E}}$

7. Regression estimators (RE)

Let $y_{1}, y_{2}, \ldots, y_{n}$ be a random sample from $\operatorname{Fr}(a, b)$. Since $\left(y_{i}\right)=e^{-a y_{i}^{-b}}$, then $\operatorname{Ln}\left(-\operatorname{Ln}\left(F\left(y_{i}\right)\right)\right)=\operatorname{Ln}(a)+$ $b \operatorname{Ln}\left(1 / y_{i}\right)$. By letting $u_{i}=\operatorname{Ln}\left(-\operatorname{Ln}\left(F\left(y_{i}\right)\right)\right), v_{i}=\operatorname{Ln}\left(1 / y_{i}\right), \gamma_{0}=\operatorname{Ln}(a)$ and $\gamma_{0}=b$ and adding iid random error (noise) $\epsilon$, one can treat with the above equation as simple linear regression model, where $u$ and $v$ are dependent and explanatory variables respectively. The regression estimators of $b$ and $a$ are as follows,

$\hat{b}_{R E}=\sum_{i=1}^{n}\left(v_{i}-\bar{v}\right) u_{i} / \sum_{i=1}^{n}\left(v_{i}-\bar{v}\right)^{2}$ and $\hat{a}_{R E}=\operatorname{Exp}\left\{\bar{u}-\hat{b}_{R E} \bar{v}\right\}$.

\section{The empirical study and discussions}

We conduct extensive simulations to compare the performances of the different methods, stated in section IV, mainly with respect to their mean square errors (MSE) for different sample sizes and for different parameters values.

Actually, there are two essential experiments, the first one was to explore the best method(s) to estimate parameters of Fréchet distribution, while the second experiment is to explore the best method(s) to estimate $R=P(X<Y)$ which is defined in (9).

The experiments were conducted according to run size $K=1000$. We reported the results for $n=10$ (small sample), $n=20$ (moderate sample) and $n=50,100$ (large sample) and for, 
1. The following different values of $a$ and $b$ in the first experiment,

\begin{tabular}{cccccc}
\hline$a$ & 0.6 & 1 & 0.9 & 1.2 & 0.3 \\
\hline$b$ & 1 & 0.6 & 0.9 & 0.3 & 1.2 \\
\hline
\end{tabular}

2. The following different values of $\mu, \alpha, \lambda$ and $\theta$ in the second experiments,

\begin{tabular}{ccccccccccc}
\hline case & $\mu$ & $\alpha$ & $\lambda$ & $\theta$ & case & $\mu$ & $\alpha$ & $\lambda$ \\
\hline 1 & 1 & 0.3 & 0.6 & 0.1 & 7 & 0.6 & 0.3 & 1 & 0.1 \\
2 & 0.1 & 0.3 & 1 & 0.6 & 8 & 0.1 & 0.3 & 0.6 & 1 \\
3 & 1 & 0.1 & 0.6 & 0.3 & 9 & 0.6 & 0.1 & 0.3 & 1 \\
4 & 0.6 & 0.1 & 1 & 0.3 & 10 & 0.3 & 0.1 & 0.6 & 1 \\
5 & 0.6 & 0.3 & 0.1 & 1 & 11 & 0.3 & 0.1 & 1 & 0.6 & 0.1 \\
6 & 1 & 0.1 & 0.3 & 0.6 & 12 & 1 & 0.3 & 0.1 & 0.6 \\
\hline
\end{tabular}

Note that, for the second experiment, $m=n$, where $m$ and $n$ are the sample sizes drawn from stress and strength variables respectively.

The results of the first experiment and the second experiment are reported in table (1) and table (2) respectively.

Some of common points are very clear from tables for both of the two experiments,

1) The MSE's decrease as sample size increases in all methods of estimation. It verifies the asymptotic unbiasedness and consistency of all the estimators.

2) It can be said that the estimation of shape parameters are more accurate for the smaller values of those parameters whereas the estimation of scale parameters are more accurate for the larger values of those parameters. in other words, MSE's increase as shape parameter increases whereas MSE's increase as scale parameter decreases.

3) The performances of RE, WLSE, LSE, EMME and AMME are according to their order.

4) The performances of RE's and WLSE's are close to each other. Also, the performances of EMME's and AMME's are close to each other.

For more detailed discussions, let us do that for each experiment,

a) For the first experiment,

For comparing the performances of all the eight methods under consideration to estimate the Parameters of Fréchet distribution, the following points can be mentioned,

i) For small $(n=10)$ sample size, it is observed that PE works the best for both of the two parameters. The performances of the LME's and MLE's are close to that of PE's. The LME's are the quite closest to PE's for the estimation of the scale parameter whereas the MLE's are the quite closest to PE's for estimation of the shape parameter.

ii) For moderate $(n=20)$ sample size, it is observed that LME works the best from all other Methods to estimate the scale parameter whereas the second and third best method is respectively, PE and MLE. The performances of PE's and MLE's are close to each other. PE works the best from all other methods to estimate the shape parameter whereas the second and third best methods are respectively, MLE and LME. The performances of MLE's and LME's are close to each other.

iii) For large $(n=50,100)$ sample size, it is observed that MLE works the best from all other Methods to estimate the scale parameter whereas the second and third best methods are respectively, LME and PE. The performances of PE's and LME's are close to each other PE works the best from all other methods to estimate the shape parameter whereas the second and third best methods are respectively, LME and MLE. The performances of MLE's and LME's are close to each other. 
Table 1: Empirical MSE to Estimate the Fréchet Distribution Parameters $a$ and $b$

\begin{tabular}{|c|c|c|c|c|c|c|c|c|c|c|c|}
\hline \multicolumn{2}{|c|}{ case } & \multicolumn{2}{|c|}{1} & \multicolumn{2}{|c|}{2} & \multicolumn{2}{|c|}{3} & \multicolumn{2}{|c|}{4} & \multicolumn{2}{|c|}{5} \\
\hline \multicolumn{2}{|c|}{ parameters } & $a$ & $b$ & $a$ & $b$ & $a$ & $b$ & $a$ & $b$ & $a$ & $b$ \\
\hline Sample size & The method & 0.6 & 1 & 1 & 0.6 & 0.9 & 0.9 & 1.2 & 0.3 & 0.3 & 1.2 \\
\hline \multirow{8}{*}{10} & MLE & 1.250 & 2.372 & 1.203 & 2.082 & 1.219 & 2.315 & 1.138 & 1.646 & 1.552 & 2.414 \\
\hline & EMME & 1.563 & 2.902 & 1.512 & 2.613 & 1.53 & 2.845 & 1.442 & 2.176 & 1.863 & 2.943 \\
\hline & AMME & 1.574 & 2.951 & 1.528 & 2.654 & 1.547 & 2.892 & 1.465 & 2.223 & 1.882 & 2.996 \\
\hline & PE & 0.982 & 2.352 & 0.936 & 2.051 & 0.954 & 2.286 & 0.862 & 1.618 & 1.287 & 2.387 \\
\hline & LSE & 1.525 & 2.878 & 1.472 & 2.576 & 1.492 & 2.809 & 1.404 & 2.142 & 1.825 & 2.914 \\
\hline & WLSE & 1.414 & 2.689 & 1.361 & 2.384 & 1.383 & 2.623 & 1.298 & 1.954 & 1.718 & 2.723 \\
\hline & LME & 1.013 & 2.385 & 0.968 & 2.082 & 0.979 & 2.323 & 0.895 & 1.658 & 1.314 & 2.426 \\
\hline & RE & 1.432 & 2.706 & 1.382 & 2.406 & 1.398 & 2.639 & 1.315 & 1.975 & 1.734 & 2.743 \\
\hline \multirow{8}{*}{20} & MLE & 0.979 & 2.333 & 0.922 & 2.036 & 0.945 & 2.267 & 0.855 & 1.598 & 1.278 & 2.365 \\
\hline & EMME & 1.536 & 2.887 & 1.481 & 2.585 & 1.502 & 2.824 & 1.412 & 2.155 & 1.836 & 2.923 \\
\hline & AMME & 1.555 & 2.928 & 1.508 & 2.622 & 1.521 & 2.866 & 1.435 & 2.194 & 1.855 & 2.963 \\
\hline & PE & 0.957 & 2.222 & 0.900 & 1.921 & 0.926 & 2.162 & 0.847 & 1.492 & 1.263 & 2.265 \\
\hline & LSE & 1.512 & 2.836 & 1.465 & 2.532 & 1.479 & 2.774 & 1.396 & 2.100 & 1.812 & 2.875 \\
\hline & WLSE & 1.413 & 2.663 & 1.363 & 2.361 & 1.379 & 2.604 & 1.295 & 1.932 & 1.714 & 2.704 \\
\hline & LME & 0.944 & 2.337 & 0.898 & 2.031 & 0.91 & 2.269 & 0.821 & 1.622 & 1.248 & 2.373 \\
\hline & RE & 1.394 & 2.692 & 1.347 & 2.398 & 1.36 & 2.631 & 1.279 & 1.966 & 1.695 & 2.734 \\
\hline \multirow{8}{*}{50} & MLE & 0.909 & 2.331 & 0.852 & 2.034 & 0.875 & 2.272 & 0.789 & 1.602 & 1.202 & 2.375 \\
\hline & EMME & 1.524 & 2.848 & 1.476 & 2.542 & 1.494 & 2.782 & 1.402 & 2.113 & 1.825 & 2.886 \\
\hline & AMME & 1.547 & 2.902 & 1.496 & 2.607 & 1.514 & 2.844 & 1.422 & 2.175 & 1.843 & 2.948 \\
\hline & PE & 0.944 & 2.187 & 0.893 & 1.882 & 0.911 & 2.124 & 0.821 & 1.458 & 1.244 & 2.225 \\
\hline & LSE & 1.427 & 2.687 & 1.378 & 2.381 & 1.396 & 2.622 & 1.314 & 1.956 & 1.732 & 2.724 \\
\hline & WLSE & 1.404 & 2.65 & 1.352 & 2.353 & 1.373 & 2.586 & 1.289 & 1.911 & 1.704 & 2.689 \\
\hline & LME & 0.936 & 2.314 & 0.881 & 2.014 & 0.902 & 2.251 & 0.812 & 1.586 & 1.236 & 2.354 \\
\hline & RE & 1.414 & 2.672 & 1.367 & 2.376 & 1.381 & 2.607 & 1.292 & 1.932 & 1.714 & 2.707 \\
\hline \multirow{8}{*}{100} & MLE & 0.873 & 2.303 & 0.824 & 2.002 & 0.839 & 2.239 & 0.751 & 1.571 & 1.173 & 2.342 \\
\hline & EMME & 1.513 & 2.766 & 1.466 & 2.467 & 1.479 & 2.701 & 1.399 & 2.032 & 1.813 & 2.803 \\
\hline & AMME & 1.535 & 2.763 & 1.484 & 2.469 & 1.561 & 2.699 & 1.416 & 2.033 & 1.838 & 2.804 \\
\hline & PE & 0.926 & 2.074 & 0.878 & 1.772 & 0.899 & 2.014 & 0.807 & 1.345 & 1.224 & 2.114 \\
\hline & LSE & 1.405 & 2.667 & 1.355 & 2.366 & 1.372 & 2.601 & 1.282 & 1.932 & 1.709 & 2.702 \\
\hline & WLSE & 1.391 & 2.636 & 1.346 & 2.334 & 1.359 & 2.569 & 1.276 & 1.922 & 1.699 & 2.673 \\
\hline & LME & 0.922 & 2.249 & 0.876 & 1.942 & 0.891 & 2.177 & 0.809 & 1.502 & 1.228 & 2.278 \\
\hline & $\mathrm{RE}$ & 1.398 & 2.647 & 1.348 & 2.341 & 1.362 & 2.582 & 1.274 & 1.913 & 1.691 & 2.683 \\
\hline
\end{tabular}

Table 2: Empirical MSE to Estimate $\mathrm{R}=\mathrm{P}(\mathrm{X}<Y)$ for the Fréchet Stress-Strength Model

\begin{tabular}{|c|c|c|c|c|c|c|c|c|c|c|c|c|c|}
\hline \multirow{2}{*}{$\begin{array}{c}\text { Sample } \\
\text { size }\end{array}$} & \multirow{2}{*}{$\begin{array}{c}\text { The } \\
\text { method }\end{array}$} & \multicolumn{12}{|c|}{ case } \\
\hline & & 1 & 2 & 3 & 4 & 5 & 6 & 7 & 8 & 9 & 10 & 11 & 12 \\
\hline \multirow{8}{*}{10} & MLE & 3.853 & 3.888 & 3.792 & 3.802 & 3.908 & 3.813 & 3.861 & 3.907 & 3.826 & 3.821 & 3.816 & 3.884 \\
\hline & EMME & 7.504 & 7.532 & 7.446 & 7.453 & 7.542 & 7.455 & 7.506 & 7.552 & 7.468 & 7.462 & 7.461 & 7.522 \\
\hline & AMME & 7.684 & 7.713 & 7.622 & 7.632 & 7.738 & 7.644 & 7.695 & 7.736 & 7.652 & 7.658 & 7.645 & 7.713 \\
\hline & PE & 3.772 & 3.807 & 3.715 & 3.729 & 3.825 & 3.722 & 3.771 & 3.824 & 3.731 & 3.748 & 3.733 & 3.792 \\
\hline & LSE & 5.278 & 5.324 & 5.216 & 5.222 & 5.312 & 5.223 & 5.276 & 5.322 & 5.231 & 5.233 & 5.231 & 5.291 \\
\hline & WLSE & 4.723 & 4.743 & 4.667 & 4.663 & 4.764 & 4.675 & 4.721 & 4.771 & 4.682 & 4.682 & 4.68 & 4.742 \\
\hline & LME & 3.727 & 3.754 & 3.668 & 3.673 & 3.762 & 3.676 & 3.722 & 3.772 & 3.682 & 3.683 & 3.681 & 3.741 \\
\hline & $\mathrm{RE}$ & 4.586 & 4.606 & 4.527 & 4.523 & 4.621 & 4.532 & 4.580 & 4.631 & 4.541 & 4.542 & 4.54 & 4.603 \\
\hline \multirow{8}{*}{20} & MLE & 3.713 & 3.742 & 3.651 & 3.661 & 3.766 & 3.676 & 3.726 & 3.767 & 3.683 & 3.688 & 3.676 & 3.746 \\
\hline & EMME & 6.965 & 6.997 & 6.908 & 6.918 & 7.018 & 6.923 & 6.975 & 7.018 & 6.935 & 6.939 & 6.927 & 6.993 \\
\hline & AMME & 7.157 & 7.176 & 7.092 & 7.097 & 7.194 & 7.101 & 7.153 & 7.201 & 7.116 & 7.112 & 7.11 & 7.172 \\
\hline & $\mathrm{PE}$ & 3.763 & 3.784 & 3.707 & 3.706 & 3.802 & 3.712 & 3.762 & 3.811 & 3.726 & 3.722 & 3.72 & 3.782 \\
\hline & LSE & 5.068 & 5.098 & 5.003 & 5.011 & 5.113 & 5.012 & 5.063 & 5.115 & 5.033 & 5.036 & 5.024 & 5.093 \\
\hline & WLSE & 4.685 & 4.717 & 4.621 & 4.633 & 4.730 & 4.642 & 4.693 & 4.736 & 4.658 & 4.652 & 4.645 & 4.716 \\
\hline & LME & 3.753 & 3.781 & 3.699 & 3.708 & 3.822 & 3.702 & 3.752 & 3.804 & 3.711 & 3.729 & 3.713 & 3.772 \\
\hline & $\mathrm{RE}$ & 4.483 & 4.500 & 4.414 & 4.422 & 4.523 & 4.434 & 4.486 & 4.529 & 4.446 & 4.442 & 4.438 & 4.508 \\
\hline \multirow{8}{*}{50} & MLE & 3.063 & 3.096 & 3.001 & 3.011 & 3.115 & 3.029 & 3.075 & 3.117 & 3.032 & 3.032 & 3.026 & 3.093 \\
\hline & EMME & 6.487 & 6.514 & 6.426 & 6.434 & 6.522 & 6.432 & 6.483 & 6.532 & 6.447 & 6.443 & 6.441 & 6.502 \\
\hline & AMME & 6.875 & 6.903 & 6.818 & 6.826 & 6.912 & 6.822 & 6.872 & 6.923 & 6.832 & 6.845 & 6.832 & 6.891 \\
\hline & PE & 3.183 & 3.216 & 3.121 & 3.132 & 3.239 & 3.133 & 3.182 & 3.235 & 3.151 & 3.152 & 3.144 & 3.216 \\
\hline & LSE & 4.612 & 4.642 & 4.558 & 4.568 & 4.654 & 4.562 & 4.611 & 4.663 & 4.573 & 4.583 & 4.572 & 4.632 \\
\hline & WLSE & 4.008 & 4.036 & 3.941 & 3.956 & 4.056 & 3.963 & 4.016 & 4.058 & 3.978 & 3.972 & 3.967 & 4.031 \\
\hline & LME & 3.244 & 3.263 & 3.173 & 3.181 & 3.284 & 3.191 & 3.247 & 3.289 & 3.206 & 3.201 & 3.198 & 3.261 \\
\hline & $\mathrm{RE}$ & 3.884 & 3.911 & 3.821 & 3.833 & 3.933 & 3.846 & 3.899 & 3.937 & 3.857 & 3.853 & 3.846 & 3.915 \\
\hline \multirow{8}{*}{100} & MLE & 2.543 & 2.563 & 2.473 & 2.481 & 2.582 & 2.499 & 2.543 & 2.589 & 2.509 & 2.502 & 2.498 & 2.566 \\
\hline & EMME & 5.176 & 5.195 & 5.116 & 5.112 & 5.211 & 5.124 & 5.171 & 5.221 & 5.132 & 5.132 & 5.13 & 5.192 \\
\hline & AMME & 5.275 & 5.308 & 5.211 & 5.227 & 5.326 & 5.234 & 5.282 & 5.326 & 5.245 & 5.246 & 5.235 & 5.307 \\
\hline & PE & 2.648 & 2.673 & 2.582 & 2.593 & 2.691 & 2.609 & 2.656 & 2.698 & 2.616 & 2.612 & 2.607 & 2.679 \\
\hline & LSE & 4.153 & 4.172 & 4.097 & 4.097 & 4.193 & 4.102 & 4.151 & 4.201 & 4.112 & 4.111 & 4.11 & 4.171 \\
\hline & WLSE & 3.225 & 3.242 & 3.156 & 3.163 & 3.260 & 3.171 & 3.229 & 3.269 & 3.189 & 3.187 & 3.178 & 3.249 \\
\hline & LME & 2.653 & 2.676 & 2.598 & 2.592 & 2.694 & 2.608 & 2.659 & 2.701 & 2.611 & 2.612 & 2.61 & 2.671 \\
\hline & $\mathrm{RE}$ & 3.176 & 3.208 & 3.113 & 3.121 & 3.221 & 3.138 & 3.185 & 3.227 & 3.147 & 3.143 & 3.136 & 3.205 \\
\hline
\end{tabular}


b) For the second experiment

b.1) the behavior effect of the shape and scale parameters is very clear on the results, as it stated in (2) of common points above. The results were amazing, since MSE's increase as the cases order increases as in the following table,

\begin{tabular}{ccccccccccccc}
\hline order & case & $\mu$ & $\alpha$ & $\lambda$ & $\theta$ & order & case & $\mu$ & $\alpha$ & $\lambda$ \\
\hline 1 & 3 & 1 & 0.1 & 0.6 & 0.3 & 7 & 1 & 1 & 0.3 & 0.6 & 0.1 \\
2 & 4 & 0.6 & 0.1 & 1 & 0.3 & 8 & 7 & 0.6 & 0.3 & 1 & 0.1 \\
3 & 6 & 1 & 0.1 & 0.3 & 0.6 & 9 & 12 & 1 & 0.3 & 0.1 & 0.6 \\
4 & 11 & 0.3 & 0.1 & 1 & 0.6 & 10 & 2 & 0.1 & 0.3 & 1 & 0.6 \\
5 & 9 & 0.6 & 0.1 & 0.3 & 1 & 11 & 5 & 0.6 & 0.3 & 0.1 & 1 \\
6 & 10 & 0.3 & 0.1 & 0.6 & 1 & 12 & 8 & 0.1 & 0.3 & 0.6 & 1 \\
\hline
\end{tabular}

b.2) for comparing the performances of all the eight methods under consideration to estimate $\mathrm{R}=\mathrm{P}(\mathrm{X}<Y)$, the following points can be mentioned,

i) For small $(n=10)$ sample size, it is observed that LME works the best for all cases. The performances of the PE's and MLE's, respectively, are quite close to that of LME's.

ii) For moderate $(n=20)$ sample size, it is observed that MLE works the best from all other Methods whereas the second and third best method are respectively, LME and PE. The performances of PE's and LME's are close to each other.

iii) For large $(n=50,100)$ sample size, it is observed that MLE works the best from all other Methods whereas the second and third best method are respectively, PE and LME. The performances of PE's and LME's are close to each other.

\section{References}

[1] Arora, P. and Singh, R. .First course in real analysis. Sultan Chand \& Sons publishers, New Delhi, India, (1972).

[2] Hosking, J. R. M. .L-Moment: Analysis and estimation of distributions using linear combinations of order statistics. Journal of Royal Statistical Society, Ser. B, (1990), 52(1), 105 - 124.

[3] Kao, J. H. K. .A graphical estimation of mixed Weibull parameters in life testing electron tubes. , Technometrics, (1959) 1,389 - 407.

[4] Mann, N. R., Schafer, R. E. and Singpurwalla, N. D. .Methods for Statistical Analysis of Reliability and Life Data. , New York, Wiley, (1974).

[5] Swain, J., Venkatraman, S. and Wilson, J. .Least squares estimation of distribution function in Johnson's translation system. , Journal of Statistical Computation and Simulation, (1988), 29, 271 - 297. 